\title{
Koebner Phenomenon in Dermatomyositis after Subcutaneous Methotrexate Injections
}

PRISCILLE COUTURE, MD, PhD, AP-HP, Hôpital Tenon, Service de Dermato-allergologie; CLAUDE BACHMEYER, MD, PhD, AP-HP, Hôpital Tenon, Service de Médecine Interne; JEAN-BENOIT MONFORT, MD, PhD, AP-HP, Hôpital Tenon, Service de Dermato-allergologie, Paris, France. Address correspondence to Dr. P. Couture, Hôpital Tenon, Service de dermatologie, UF de dermatologie vasculaire, 4, rue de la Chine, 75970 Paris, Cedex 20, France. E-mail: couture.priscille@gmail.com. Ethics approval was not required for a case report according to French institutional protocol. This article complies with the requirements of the amended law of January 6, 1978. This study is strictly in accordance with the rules set out in the reference text MR3. The article was registered on the CNIL Website and received the CERFA number $13810 * 01$ of the compliance commitment form. The patient's written informed consent was obtained to publish the material. J Rheumatol 2019;46:1350; doi:10.3899/jrheum.181188

Koebner phenomenon has rarely been reported in patients with dermatomyositis, in one case from steam burn ${ }^{1}$ and in another, a possible scratch ${ }^{2}$.

A 71-year-old woman sought medical advice for 3 erythematous patches on her thighs that had developed after subcutaneous methotrexate injections (Figures 1A and 1B). Six months before, she had presented typical lesions of dermatomyositis including heliotrope rash, eyelid erythema, and Gottron papules but no muscle manifestations. A skin biopsy from the back had shown interface dermatitis. A diagnosis of paraneoplastic amyopathic dermatomyositis associated with metastatic ovarian cancer had been established. The cancer had been previously treated by surgery and chemotherapy and was considered in remission.

Treatment with topical corticosteroid and hydroxychloroquine was ineffective. Therefore, methotrexate $(20 \mathrm{mg}$ per week) was given subcutaneously on the thighs. Two days after each injection, the patient complained of an erythematous lesion at the injection site that was still present when the patient eventually came to consult. In the same time, the initial dermatomyositis lesions had improved.

A skin biopsy from the last injection site revealed an interface dermatitis (Figure 1C). The development of clinical lesions with consistent histological pattern of dermatomyositis following trauma in a previously unaffected area is suggestive of Koebner phenomenon. This is an isomorphic response observed in many dermatoses such as psoriasis.

\section{REFERENCES}

1. Ng PP, Gan SL. Koebner phenomenon in dermatomyositis. Dermatology 2006;213:249-50.

2. Tidwell WJ, Callen JP. Koebner phenomenon in juvenile dermatomyositis. J Rheumatol 2018;45:437.
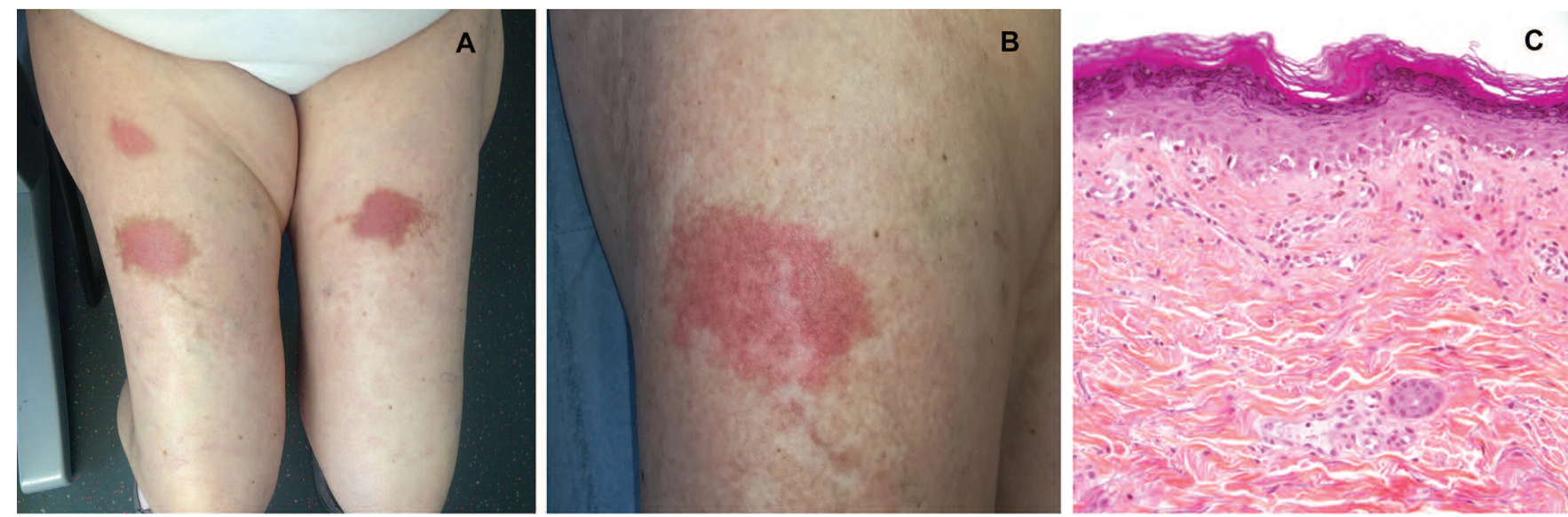

Figure 1.A, B. Erythematous patches on thighs that had developed after subcutaneous methotrexate injections. C. Interface dermatitis revealed by skin biopsy from the last injection site. 